\title{
Social Skins of the Head: Body Beliefs and Ritual in Ancient Mesoamerica and the Andes Edited by V. Tiesler and M. C. Lozada (2018) Albuquerque: University of New Mexico Press, xv + 283pp.
}

\author{
Reviewed by Sara L. Juengst
}

Keywords: CRANIA; RITUAL; ANDES; MeSOAMERICA; IDENTITY

Researchers have long recognized the symbolic and spiritual significance of the human head across time and space. Archaeological and bio-archaeological works often show that the head in the Americas was considered to be a locus of personhood and the soul, an embodied site of ancestral identity, and a potent symbol of human and earthen fertility, in both living and deceased persons. This edited volume tackles the use (and reuse) of human heads and head imagery in Mesoamerica and the Andes, looking at common themes and distinct uses over time in these regions.

Heads are both biologically vital and individually recognizable, making them potent symbols of identity of both the living and the dead. Furthermore, once removed from a human body, a head can be easily manipulated as a cultural artifact. Thus, heads have often been used as mortuary offerings, war trophies, representations of individuals, and indicators of collective identity. In the Andes and Mesoamerica, heads of the living and deceased were regularly manipulated to convey messages of belonging, status, ethnicity, fertility, dominance, and control. Modified crania, face-painting, and hairstyles gave symbolic power to and identified living individuals, with varying degrees of permanence. In mortuary contexts, isolated crania played many roles, from demonstrating military and physical

\section{Affiliation}

University of North Carolina, Charlotte, USA.

email: sjuengst@uncc.edu 
prowess over conquered victims, to representing seeds and regeneration, created from kin and enemies alike.

This volume is divided regionally, focusing first on Mesoamerica (and northern Mexico in particular) and then on Andean South America (Peru and Bolivia). The authors use mixed methods to investigate heads in the past and present, integrating interpretations of physical skeletal remains, linguistic clues, and iconographic representations. The chapters investigate two main themes: the symbolism of heads during life (how humans manipulated their own heads or the heads of their children) and the afterlives of heads (how heads were treated after death).

In both Mesoamerica and Andean South America, the heads of living individuals were manipulated, in order to convey messages of power and belonging. This took a variety of forms, from the physical molding of the skull into various desired shapes during infancy and childhood, to face decoration in the form of paint or tattooing, to hairstyles conveying occupation and status. Artificial cranial modification is the most permanent of these manipulations, and imposed an identity on the very young, as this procedure must be done during infancy or early childhood while the cranial bones are still forming. Reasons for modifying crania are as diverse as the styles of modification themselves. Duncan and Vail (Chapter 2), Scherer (Chapter 4), and Blom and Couture (Chapter 13) demonstrate the necessity of cranial binding for the ritual protection of the soul and development of the adult individual in both Mesoamerica and the Andes. Simultaneously, Tiesler and Lacadena (Chapter 3), Alvarado-Viñas and Manzanilla (Chapter 5), and Mannheim, Davis and Velasco (Chapter 14) show how Mesoamerican and Andean ethnic identities were communicated through style of cranial modification, especially important in frontier areas, multiethnic communities, or hierarchical societies. In all cases, modified crania had tiered meanings: individual protection, ties to familial groups, and ethnic or status implications.

Face painting and hairstyles were also important signifiers of identity. In Chapter 6, Vázquez de Ágredos Pascual, Lorenzo and Campos catalog known instances of face painting (or tattooing) visible on Maya murals, statues, and ceramics. These authors demonstrate how facial treatments conveyed important information regarding the function of an individual in the scene, rather than an inherent social identity such as gender. In other words, face painting was highly contextual and patterns of adornment were not exclusive to certain segments of society. Similarly, hairstyles probably represented classes of people and their role in the particular scene. Thus, the focus here was the purpose of heads in relation to the broader Maya universe, not the individual. This is an important finding, as much scholarship

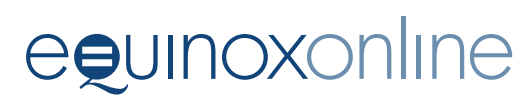


focuses on identifying particular individuals from facial features. While this is at times appropriate (such as in Chapter 7 of this volume), the idea of collective identities in the past cannot be underestimated. This is underscored in Chapter 16 by interpretations of Wari ritual vessels from Peru. Vazquez de Arthur convincingly demonstrates that 'faceneck' jars (large ceramics with stylized faces molded onto the necks of the vessels) were portraits of shared identities, rather than distinct individuals.

Heads did not lose their symbolic meaning after death; in fact, many of these chapters focus on what heads isolated from bodies signified for Mesoamerican and Andean peoples. In many ways, these heads had lives, or as Verano (Chapter 11) terms it, 'afterlives', of their own, manipulated by friends and foes alike. Broadly, disarticulated or isolated heads can be classified as (1) trophy heads captured in battle and meant to demonstrate power over a foe, (2) heads of venerated ancestors, or (3) deviant or powerful individuals who needed to be controlled. Notably, these categories are not mutually exclusive. Chávez Balderas (Chapter 9), Verano (Chapter 11), and Cecilia Lozada and colleagues (Chapter 12) make compelling arguments about isolated crania from Mexico and the coast of Peru. These heads were modified, used, and reused for various reasons, but across all examples, warfare and violent conflict played a central role in how individual heads became isolated from bodies. Trophy heads and decapitated crania demonstrated physical prowess over others, and the display of these disembodied heads for both Mesoamericans and Andeans sent powerful messages about power and control of human and spiritual realms.

Blom and Couture (Chapter 13) and Hastorf (Chapter 17) discuss how isolated heads were necessary for promoting and protecting livelihoods. Heads at times acted as 'seeds,' buried or curated in ritual ways to ensure fertility for humans, animals, and plants alike. In fact, protecting and/ or curating the heads of important members of society may have been paramount for social security, as demonstrated by the retrieval and burial of crania at La Ramada, as discussed by Cecilia Lozada and associates (Chapter 12). Conversely, controlling the crania of those countering the empowered or deviant from society was also important. The mutilated crania presented by Becker and Alconini (Chapter 15) are a stunning example of controlling deviants, as these individuals probably had their eyes and mouths removed, effectively silencing these individuals in life and death. The manipulation of heads after death clearly sent messages about individual and collective power, just as in life.

This volume weaves together multiple lines of evidence from many parts of Mesoamerica and the Andes to effectively demonstrate the importance of heads for diverse peoples. While no two cultures used heads or head

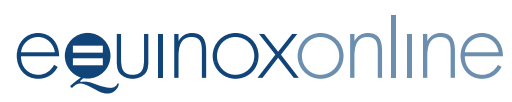


imagery in the same way, the themes around identity and power clearly resonate across Central and South America. Drawing together studies of both living and dead crania is an important contribution to archaeology and anthropology more broadly, as this volume shows how the head is not just biologically vital, but is woven into the fiber of past (and present) societies. One interesting avenue not explored by this volume is the use of animal heads alongside human ones. If we identify the human head as the locus of social identity in many cases, how do we interpret the use of isolated animal heads for these groups? Comparisons in the use of human and animal heads may reveal interesting insights about what it meant to be human in Mesoamerica or the Andes in the past. Additionally, expanding these studies to other areas of Central and South America would likely simultaneously reveal more variation and continuity in the centrality of the head as focus for symbolism and ritual.

Overall, Social Skins of the Head is a great multi-method investigation of the social importance of heads in Mesoamerica and the Andes. Drawing on ethnography, linguistics, and iconography, the authors integrate archaeological and bio-archaeological data on heads into broader data sets, contextualizing how heads are used in that particular instance. The resulting volume is a powerful work that highlights themes concerning the power of heads and identity, which resonate across the Americas. 